examined focusing on the age, CCI at hospitalization, Karnofsky Performance Status (KPS), surgery, radiotherapy (standard and hypofractionated), temozolomide-based chemotherapy. RESULTS: There was no significant correlation between age and CCI, but a weak negative correlation between KPS and CCI $(r=-0.302)$. Mean OS was 13.5 months in the resection group, $8.6 \mathrm{~m}$ in the biopsy group and 9.2 months in the non-surgery group. OS was longer in the resection group, but no difference in CCI. No significant difference in OS between standard radiotherapy with TMZ and hypofractionated radiotherapy with TMZ (13.2 m vs $11.5 \mathrm{~m})$, with no concern to CCI. OS tended to be shorter in the high CCI group ( $8.6 \mathrm{~m}$ vs $14.5 \mathrm{~m}$ ). DISCUSSION: The presence of comorbidities is one of the factors related to prognosis, and glioblastoma affecting the brain is in a disadvantageous condition due to cognitive dysfunction and paralysis. Since surgical excision and radiation chemotherapy lead to improvement in prognosis even in the elderly, it is important to complete the treatment paying maximum attention to the management of comorbidities. CCI would be useful to evaluate comorbidities. Further examination is needed in large cohort.

\section{HOUT-04. COMPARISON OF ENDOSCOPIC AND OPEN SKULL BASE SURGICAL RESECTION OF HIGH-GRADE ESTHESIONEUROBLASTOMA OVER THE LAST DECADE: THE UC IRVINE EXPERIENCE}

Ronald Sahyouni, Frank PK Hsu and Gilbert Cadena; University of California, Irvine, Irvine, CA, USA

BACKGROUND: Esthesioneuroblastoma (ENB) is a rare malignant neoplasm that can be treated with open or endoscopic skull base approaches. We compared both approaches and present our outcome data. METHODS: A retrospective review from 2007 through 2017 yielded 12 ENB patients. Kadish and TNM stage, Hyams grade, Ki-67, adjuvant chemoradiation, and resection type were correlated with survival, recurrence and surgical morbidity. RESULTS: Mean age was $61.1 \pm 9.4$ years, $25 \%$ of patients were female. Median follow-up was 4.6 years. $8 \%, 67 \%$, and $25 \%$ of patients were Kadish stage B, C, and D, respectively. Hyams grade III and IV tumors were equally distributed among both groups. Eleven patients underwent surgical resection; five underwent endoscopic, five underwent open, and one patient underwent combined resection. In the endoscopic group, four patients were Kadish stage C, compared to three with Kadish stage D in the open group. TNM in the open group was uniformly T4N1M0, while the endoscopic group was T1-2N0M0. Percentage of tumors with Ki-67 >15\% was equal in both groups. Positive margins were identified in $60 \%$ of endoscopic resections and $20 \%$ of open resections. Five patients had postoperative complications, including $20 \%$ of endoscopic patients and $60 \%$ of open patients. Eleven patients received radiotherapy, and $67 \%$ of patients received chemotherapy that included cisplatin/etoposide. $50 \%$ of patients were disease-free at last follow up. $80 \%$ of patients who had open resection experienced local recurrence, compared to $0 \%$ in the endoscopic group. Median time to recurrence in the open group was 4 years, and 4.4 years across all patients, with $60 \%$ of recurrences occurring $>5$ years from the initial surgery. CONCLUSIONS: Our data suggests that endoscopic resection of high grade ENB (Kadish C) with adjuvant chemoradiation can have favorable results with fewer postoperative complications compared with open resection.

\section{HOUT-05. THE ELEVATED PRETREATMENT ASPARTATE AMINOTRANSFERASE TO LYMPHOCYTE RATIO INDEX PREDICTS POOR PROGNOSIS IN PATIENTS WITH NEWLY DIAGNOSED GLIOBLASTOMA MULTIFORME}

Jie Zhang, Zhenqiang He and Yong Gao Mou; Sun Yat-sen University

Cancer Center, Guangzhou, China

BACKGROUND: This study aimed to determine the prognostic value of pretreatment aspartate aminotransferase to lymphocyte ratio index (ALRI) in patients with newly diagnosed glioblastoma multiforme (GBM) treated with surgery as well as adjuvant radiotherapy and chemotherapy. METHODS: We retrospectively analyzed 91 newly diagnosed GBM patients treated at Sun Yat-sen University Cancer Center between June 2001 and July 2014 The optimal cut-off value of ALRI was determined using Receiver operating characteristic (ROC) curve analysis. Univariate and multivariate analysis using cox regression model was applied to evaluate the independent prognostic value of ALRI for overall survival (OS) and progression-free surviva (PFS) in GBM patients. RESULTS: The optimal cut-off value of ALRI was $6.928\left(\mathrm{U} / 10^{9}\right)$. No significant correlation between ALRI and other clinicopathological parameters was observed. The Kaplan-Meier analysis demonstrated that both PFS $(\mathrm{p}=0.01)$ and OS $(\mathrm{p}=0.002)$ were significantly worse in GBM patients with high ALRI level. Multivariate analysis further demonstrated ALRI as a predictive factor for both PFS (HR: 2.010, 95\% CI: $1.068-3.748, \mathrm{p}=0.031$ ) and OS (HR: $2.667,95 \% \mathrm{CI}: 1.262-5.634, \mathrm{p}=0.01$ ) independent of age and extent of resection. CONCLUSIONS: The result suggested that the pretreatment ALRI may be a simple and useful prognostic marker for GBM patients.
HOUT-06. TTFIELDS FOR NEWLY DIAGNOSED GLIOBLASTOMA: IMPACT OF CONSULTATION STRATEGY

Martin Proescholdt ${ }^{1,2}$, Julia Onken $^{3}$, Peter Hau ${ }^{1,4}$, Christian Doenitz ${ }^{1,2}$ and Martin Misch $^{3} ;{ }^{1}$ Wilhelm Sander-NeuroOncology Unit, University Regensburg Medical Center, Regensburg, Germany, ${ }^{2}$ Department of Neurosurgery, University of Regensburg, Regensburg, Germany, ${ }^{3}$ Department of Neurosurgery, Charité-Universitaetsmedizin, Berlin, Germany, ${ }^{4}$ Neurology / Neurooncology, University of Regensburg, Regensburg, Germany

INTRODUCTION: After a positive randomized clinical trial showing significant survival benefits, Tumor Treating Fields (TTFields) have been implemented in the management of newly diagnosed glioblastoma (GBM). However, this treatment critically depends on patient's motivation for this therapy plus a high compliance rate. Hair shaving, skin care, application of the arrays and carrying the power source in daily life requires an adequate level of commitment from the patients and their relatives. Based on our clinical experience, it became evident that structure and didactics of the initial and follow up consultations profoundly influence these parameters. METHODS: In the primary time segment of 4 months after publication of the EF-14 trial results, we have informed our patients during inpatient stay shortly after resection and histological diagnosis about this treatment option. After initially unsatisfactory experiences regarding patient motivation for TTFields treatment, we have initiated a designated outpatient clinic for TTFields therapy consultation in analogy to consultation for radiation and chemotherapy. In this setting, we allow about one hour for each patient appointment to meet the need for information about mode of action, clinical efficacy, compliance aspects as well as practical issues with the device application. We developed a standardized patient education concept for intense and open communication about the disease, prognosis and treatment options with advantages and drawbacks. RESULTS: Using the initial consultation strategy, we were unable to motivate any patients with newly diagnosed GBM to get TTFields treatment. In contrast, after changing the setting into a more patient - centered approach, and standardization of the consultation didactics, $68 \%$ of all newly diagnosed patients agree to apply TTFields therapy. In addition, the average compliance rate was well above $90 \%$, which ensures clinical efficacy of this treatment modality. CONCLUSION: Motivation for and compliance to TTFields therapy may critically depend on the consultation setting and strategy.

HOUT-07. PROTON THERAPY PATTERNS OF CARE AMONG PEDIATRIC AND ADULT PATIENTS WITH CNS TUMORS Yolanda Tseng ${ }^{1,2}$, William Hartsell ${ }^{3}$, Henry Tsai ${ }^{4}$, Shahed Badiyan ${ }^{5}$, Lane Rosen $^{6}$, Carl Rossi ${ }^{7}$, Sujay Vora ${ }^{8}$, Carlos Vargas ${ }^{8}$, Gary Larson ${ }^{9}$ and Lia Halasz ${ }^{1,2}$; ${ }^{1}$ Department of Radiation Oncology, University of Washington, Seattle, WA, USA, ${ }^{2}$ SCCA Proton Therapy Center, Seattle, WA, USA, ${ }^{3}$ Northwestern Medicine Chicago Proton Center, Chicago, IL, USA, ${ }^{4}$ ProCure Proton Therapy Center New Jersey, Somerset, NJ, USA, ${ }^{5}$ University of Maryland, Baltimore, MD, USA, ${ }^{6}$ LSU Health Science Center Shreveport, Shreveport, LA, USA, ${ }^{7}$ Scripps Proton Therapy Center, San Diego, CA, USA, ${ }^{8}$ Mayo Clinic Arizona, Scottsdale, AZ, USA, ${ }^{9}$ ProCure Proton Therapy Center Oklahoma, Oklahoma City, OK, USA

BACKGROUND: Proton therapy (PRT) is associated with reduced integral dose to normal brain tissue. While preliminary data is evolving on the efficacy of PRT for CNS tumors, little is known regarding which CNS patients are treated with PRT. We hypothesized that a large proportion of brain patients treated with PRT are young (pediatrics: <=18 years, young adults: $19-40$ years) given the longer life expectancy over which they are at risk for CNS toxicity. METHODS: We utilized the Proton Collaborative Group (PCG) registry, which prospectively follows patients across 8 academic and community PRT centers in the United States. Patients with spine tumors were excluded. The relative proportion of various histological classifications were evaluated by age group. RESULTS: Between 2009 and 2017, 1295 patients with diagnosis of a primary brain tumor were treated with PRT. Median age at PRT was 30.7 years (range, 0.73-92.2) with relatively equal sex distribution ( $52.5 \%$ male, $47.4 \%$ female). Most patients were pediatric $(37.8 \%)$ or young adult $(22.2 \%)$ and received PRT at initial diagnosis $(67.3 \%)$ rather than at recurrence $(32 \%)$. The frequency of tumor types treated varied by age group. Pediatric patients received PRT most frequently for embryonal tumors $(28.2 \%)$, astrocytic tumors $(19.8 \%)$, and ependymal tumors $(19.4 \%)$. Among young adults, astrocytic tumors $(27.4 \%)$, oligodendroglial/mixed gliomas $(17.4 \%)$, and embryonal tumors $(12.2 \%)$ were most frequently treated. Last, among adults $>40$ years, meningeal tumors $(35.8 \%)$ were most commonly treated with PRT, followed by astrocytic tumors $(27.1 \%)$ and oligodendroglial/ mixed gliomas $(11.4 \%)$. CONCLUSIONS: Primary brain tumor patients treated with PRT are young, with the majority treated for relatively favorable tumor types. This likely reflects the selection of patients that may derive the greatest benefit from reduction in brain dose. Additional studies including comparison of tumor types among CNS patients treated with photons are warranted. 